\title{
Tailoring neoadjuvant chemotherapy for patients with breast cancer who have achieved pathologic complete response
}

\author{
Xianjun Li", Yang Liu", Ming Shan, Bingqi Xu, Yubo Lu, Guoqiang Zhang \\ Department of Breast Surgery, Harbin Medical University Cancer Hospital, China \\ Contributions: (I) Conception and design: X Li, G Zhang; (II) Administrative support: G Zhang; (III) Provision of study materials or patients: G \\ Zhang; (IV) Collection and assembly of data: X Li, B Xu, M Shan, Y Lu; (V) Data analysis and interpretation: X Li, L Yang, B Xu; (VI) Manuscript \\ writing: All authors; (VII) Final approval of manuscript: All authors. \\ \#These authors contributed equally to this work. \\ Correspondence to: Guoqiang Zhang. Department of Breast Surgery, Harbin Medical University Cancer Hospital, 150 Haping Road, Harbin 150081, \\ China. Email: 6-fu@163.com.
}

Background: We retrospectively examined whether different cycles of chemotherapy affected the prognosis of patients who achieved a pathologic complete response (pCR).

Methods: We reviewed data from patients who achieved pCR after neoadjuvant chemotherapy (NACT) between 2008 and 2018. In total, 286 patients were divided into three groups: group one (n=148, 52\%) completed standard chemotherapy cycles before surgery, group two (n=81, 28\%) did not complete standard chemotherapy cycles before surgery or received chemotherapy after surgery, and group three $(n=57$, $20 \%$ ) did not complete standard chemotherapy cycles before surgery but completed them after surgery. Recurrence-free survival (RFS) was estimated using the Kaplan-Meier method, and differences between groups were evaluated by the log-rank test. Cox proportional hazards regression analysis was adjusted for different NACT groups, age, Ki-67 levels, and clinical stages.

Results: After a median follow-up of 26 months, there were no significant differences in RFS among the NACT groups $(\mathrm{P}=0.14)$. Multivariate analysis showed that $\mathrm{Ki}-67 \geq 40 \%(\mathrm{P}=0.03)$ and clinical stage (IIIB + IIIC) $(\mathrm{P}=0.002)$ might be risk factors for recurrence in patients with pCR. There were no significant differences in survival among subgroups according to Ki-67 levels and clinical stages.

Conclusions: Our study suggests that, even with pCR, patients with baseline stage IIIB or IIIC or Ki-67 levels $\geq 40 \%$ may have an increased risk of recurrence. The RFS of patients with pCR was not associated with the completion of standard chemotherapy cycles, even in high-risk patients. Therefore, the prevention of excessive chemotherapeutic treatment by de-escalation is necessary for patients with pCR.

Keywords: Breast cancer; neoadjuvant chemotherapy (NACT); de-escalation of chemotherapy; survival after pathologic complete response (survival after pCR)

Submitted Sep 17, 2019. Accepted for publication Dec 26, 2019.

doi: $10.21037 /$ tcr.2020.01.01

View this article at: http://dx.doi.org/10.21037/tcr.2020.01.01

\section{Introduction}

Neoadjuvant chemotherapy (NACT), initially used to downstage breast cancer, may achieve pathologic complete response (pCR) in patients with no residual invasive breast or axillary disease (1). An increasing number of indications for NACT have been recently described; in addition to downstaging, NACT may benefit patients with human epidermal growth factor receptor (HER)2positive or triple-negative stage II-III breast cancer and hormone receptor-positive (HR+) patients with an explicit need for chemotherapy (2). NACT did not improve survival in patients with similar baseline characteristics and chemotherapy regimens when compared with adjuvant 
chemotherapy (3). However, residual cancer burden has been demonstrated as a useful predictor of survival after NACT $(4,5)$, especially in patients with triple-negative or HER2-positive breast cancer; thus, patients who have not achieved pCR could benefit significantly from intensive postoperative adjuvant chemotherapy $(6,7)$. The prognosis of patients with pCR is good, and additional adjuvant chemotherapy may not confer survival benefits of clinical or statistical significance. Therefore, de-escalation of chemotherapy is essential in these patients to reduce unnecessary toxicity and cost and avoid excessive treatment (8).

We aimed to evaluate if the prognoses of patients who achieved pCR differed between those who completed a standard NACT regimen and those who did not. We also wished to assess whether it is appropriate for all patients with the end goal of pCR to receive the same course of NACT and whether de-escalation of NACT for patients with risk factors for recurrence after pCR is appropriate. To date, no studies have demonstrated the appropriate timing for terminating chemotherapy in patients who achieve pCR during NACT. Previous studies have suggested that different molecular types and NACT regimens may not affect the prognosis of patients who achieve pCR $(1,9)$; however, image-guided biopsy during the course of NACT can predict pCR $(10,11)$. Therefore, the primary objectives of this study were to evaluate the influence of a reduced number of cycles of the standard NACT regimen on the prognosis of patients with pCR and to identify independent risk factors for recurrence among these patients.

\section{Methods}

Between 2008 and 2018, the records of patients who were diagnosed with primary breast cancer by histopathology and had achieved pCR after NACT were retrieved from the database of Harbin Medical University Cancer Hospital. pCR was defined in this study as the eradication of invasive disease from both breast and lymph nodes (ypT0/Tis, ypN0) (1). Tumors were classified according to the 2013 St. Gallen surrogate definition of intrinsic subtypes (12). Patients were excluded if they met any of the following criteria: (I) those who received any part of chemotherapy or surgery at outside institutions; (II) those who had already completed standard cycles of NACT supplemented with additional chemotherapy; (III) those with bilateral breast cancer; (IV) those with stage IV disease when diagnosed; (V) those with the Luminal-A molecular subtype; (VI) those treated without taxanes; (VII) those with non-invasive ductal carcinoma; (VIII) those for whom no follow-up information was available.

The NACT grouping of Harbin Medical University Cancer Hospital is shown in Figure 1. Patients were divided into the following three groups: group one, patients who completed all cycles of a standard regimen of NACT before surgery and achieved pCR; group two, those who did not complete all cycles of a standard NACT regimen before or after surgery; group three, those who did not complete all cycles of a standard NACT regimen before surgery but completed the regimen after surgery. The standard NACT regimen is: 8 cycles of doxorubicin and cyclophosphamide followed by docetaxel (AC-T) (H), 6 cycles of docetaxel, doxorubicin, and cyclophosphamide (TAC) (H), 12 weeks of paclitaxel plus carboplatin $(\mathrm{PCb})(\mathrm{H})$, and either 4 cycles of epirubicin and cyclophosphamide (EC) or 18 weeks of $\mathrm{PCb}(\mathrm{H})(13)$. The recurrence-free survival (RFS) of patients with pCR was compared among the three groups and defined as the time from diagnosis of breast cancer to the occurrence of regional or distant recurrence or death of the patients.

The Kaplan-Meier method was used to estimate RFS. First, univariate analysis was performed, and differences among the three groups were evaluated by the log-rank test. A P value $<0.05$ was represented statistical significance. Significant factors identified by univariate analysis were analyzed using a Cox proportional hazards regression model and adjusted for different NACT groups (reference, group one), age (reference, age $>60$ years), clinical staging (reference, stage IIA and IIB), and Ki-67 levels (reference, Ki-67 $<40 \%)$. Differences in survival among the three groups were compared in reference to the high-risk factors identified by Cox proportional hazard regression analysis.

\section{Results}

\section{Patients}

Between 2008 and 2018, a total of 340 patients achieved pCR after NACT at our hospital, and 54 were excluded by the exclusion criteria. The remaining 286 patients underwent survival analysis (Figure 2).

All 286 patients underwent radical surgery and were divided into three different NACT groups. Group one comprised 148 patients (52\%), group two comprised 81 patients (28\%), and group three comprised 57 patients (20\%). Details of patient characteristics are shown in Table 1 . Most patients had stage IIIA disease ( $\mathrm{n}=128,45 \%)$, and 70 
Assessment of treatment efficacy of NACT patients

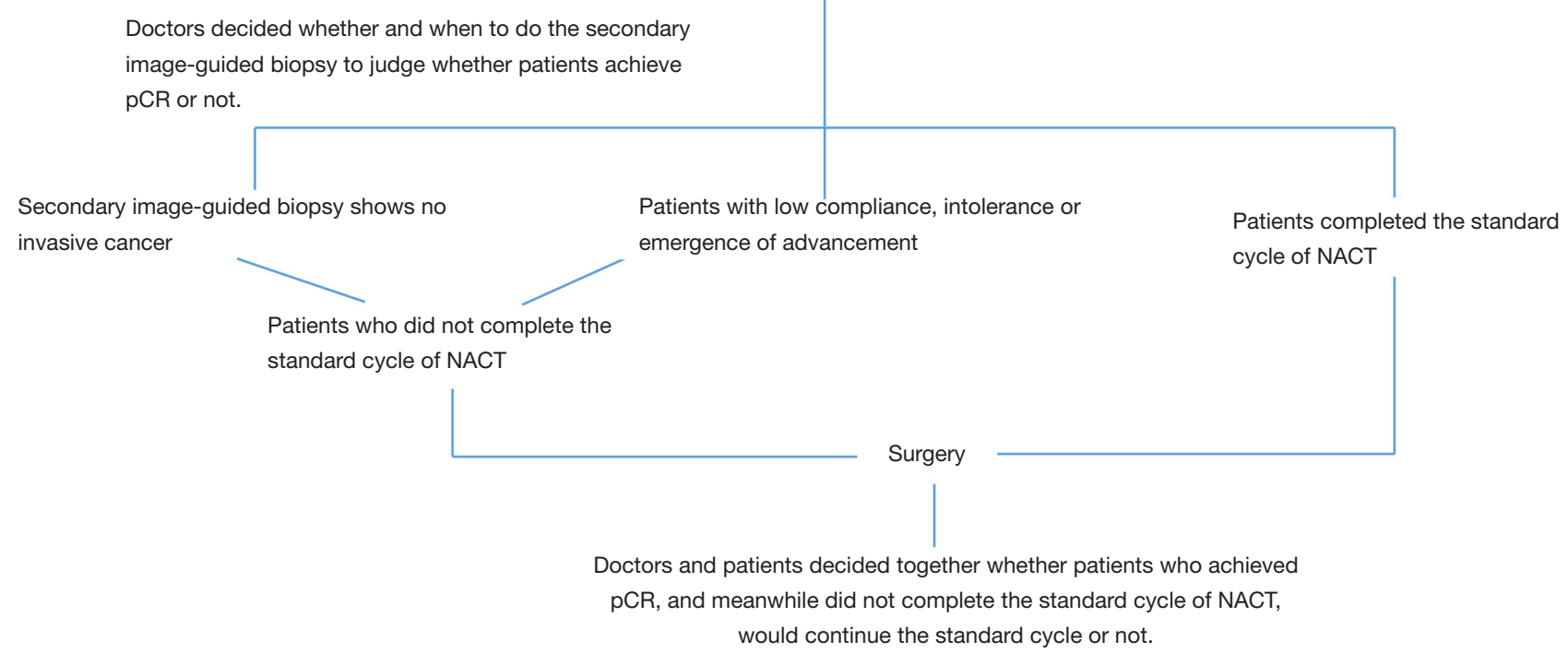

Figure 1 NACT Groups of Harbin Medical University Cancer Hospital. NACT, neoadjuvant chemotherapy; pCR, pathologic complete response.

patients (24\%) had stage IIIB and IIIC disease. There were 96 (34\%) patients whose Ki-67 levels were $\geq 40 \%$. The most common NACT regimen was A $+\mathrm{T}(\mathrm{n}=218,76 \%)$. There were 85 HER $2+$ patients (63.9\%) who did not receive HER2-targeted therapies. Anthracyclines and taxanes were administered in combination for most patients ( $\mathrm{n}=170$, $59 \%)$. Postoperative adjuvant endocrine therapy was administered to 65 patients (23\%), and 77 patients (27\%) received postoperative adjuvant radiation therapy. Table 1 summarizes the therapeutic regimens.

\section{Survival analysis}

The median follow-up time was 26 (range, 3-135) months, and the mean follow-up time was 38.8 months. There were 38 recurrence or death events: 35 patients had disease progression and 3 patients died without recurrence. Of the patients with recurrence, $23(61 \%)$ had recurrences at a distant site first, of which there were 10 (26\%) brain, 5 (13\%) bone, $2(5 \%)$ liver, and 5 (13\%) lung metastases (Table 1).

There was no statistically significant difference in RFS among the three groups $(\mathrm{P}=0.14)$ (Figure 3$)$. The 3 -year RFS was approximately $88 \%$ in group one and approximately $90 \%$ in groups two and three. Univariate analysis showed an association between clinicopathological features and survival (Table 2). Age $(\mathrm{P}=0.33)$, menstrual status $(\mathrm{P}=0.76)$, molecular subtype $(\mathrm{P}=0.10)$, Ki-67 level $(\mathrm{P}=0.069)$, NACT regimen $(\mathrm{P}=0.75)$, sequence of administration $(\mathrm{P}=0.28)$, administration schedule $(\mathrm{P}=0.90)$, adjuvant endocrine therapy $(\mathrm{P}=0.90)$, and adjuvant radiation therapy $(\mathrm{P}=0.36)$ had no significant association with recurrence in patients with pCR. However, clinical stage was significantly associated with RFS $(\mathrm{P}<0.0001)$.

We summarized the possible independent prediction factors of patients who had end-point events. Adjusted RFS hazard ratios (HRs) of death, based on patient characteristics and treatments received, are shown in Table 3. Compared with the patients with stage II, stage IIIA did not significantly increase the HR of RFS $(\mathrm{P}=0.28)$, and patients with end-point events showed no statistically significant difference $(\mathrm{P}=0.28)$. However, patients with stages IIIB and IIIC had a significantly higher number of recurrence events than did patients with stage II, and stage IIIB and IIIC disease were identified as an independent prognostic factor for RFS [HR, 5.31; 95\% confidence interval (CI), 1.64-15.44; $\mathrm{P}=0.002]$. Ki-67 levels $\geq 40 \%$ was also identified as an independent prognostic factor for RFS (HR, 2.91; 95\% CI, 1.09-7.80; $\mathrm{P}=0.03)$. We further compared the RFS of patients from different NACT groups within subgroups according to clinical staging and Ki-67 levels (Figure 3). There were no significant statistical differences among the three groups in not only patients with stage II and IIIA 


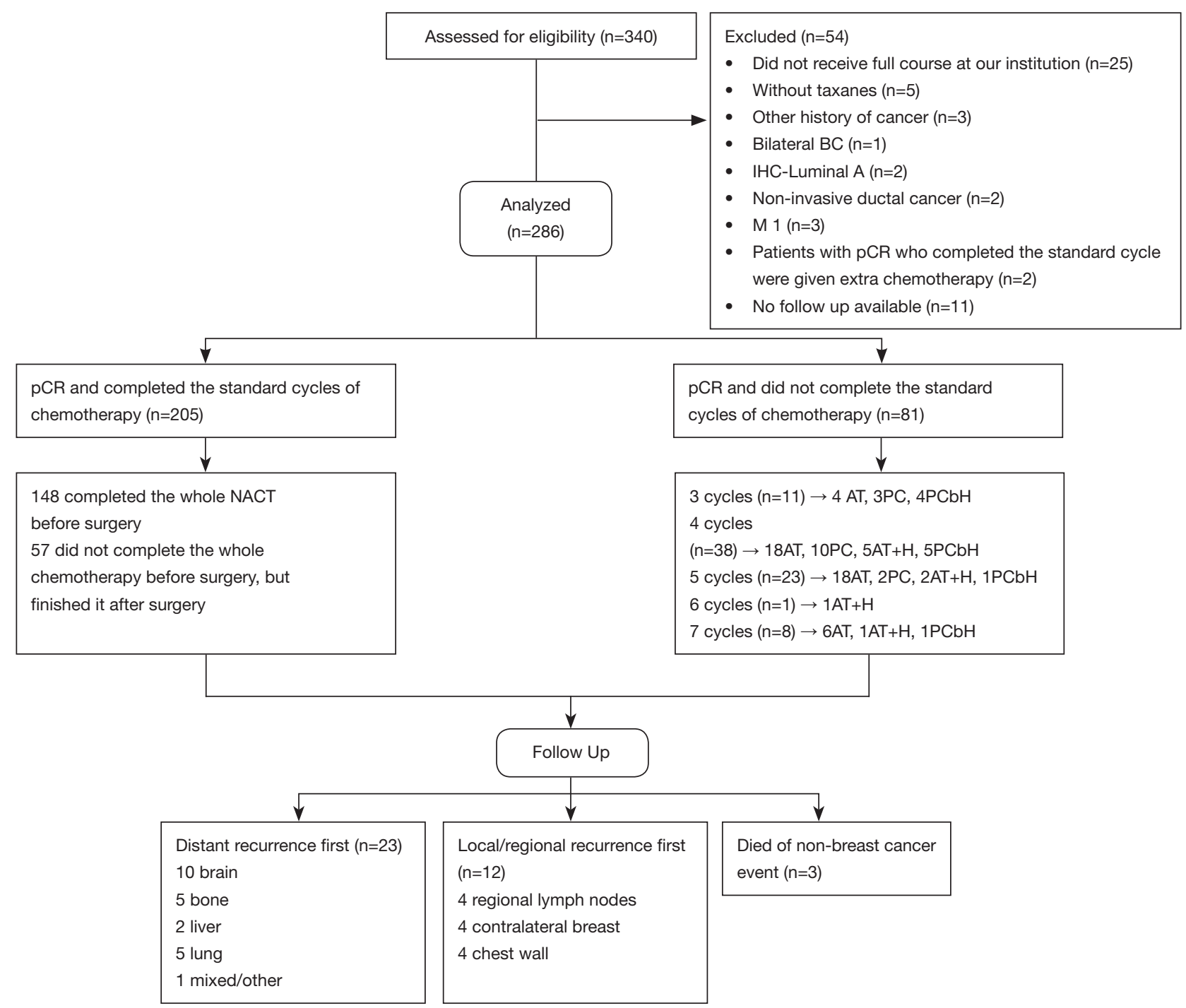

Figure 2 CONSORT diagram. CONSORT, Consolidated Standards of Reporting Trials; NACT, neoadjuvant chemotherapy; pCR, pathologic complete response; AT, adriamycin plus taxane; $\mathrm{PCb}$, paclitaxel plus carboplatin.

disease but also those at a "high risk" of recurrence (stage IIIB, IIIC and Ki-67 $\geq 40 \%$ ) (Figure 4).

\section{Discussion}

Our study did not observe clinically or statistically significant improvement in the survival of patients with pCR after NACT; therefore, further adjuvant chemotherapy may be unnecessary for these patients. In the past, adjuvant chemotherapy was prescribed after only evaluating regional lymph nodes as the main prognostic factor, with patients and doctors alike focusing on small statistical differences in survival curves (14). This resulted in the overtreatment of many patients. In contrast, we believe chemotherapy for patients with breast cancer should be based on a comprehensive assessment of the risk of recurrence, chemosensitivity, risk of treatment, and economic cost rather than a list of currently available chemotherapy regimens (15). In the absence of useful tumor markers, the benefits of additional chemotherapy are already meager (16). Additional chemotherapy benefits a minority of patients, whereas the majority receive no benefit. However, NACT with the primary goal of pCR is the gateway to individualized treatment for patients with 
Table 1 Patient characteristics of the entire cohort

\begin{tabular}{|c|c|c|}
\hline Characteristics & Number of patients & $\%$ \\
\hline \multicolumn{3}{|l|}{ Age, years } \\
\hline $21-40$ & 58 & 20 \\
\hline $40-60$ & 205 & 72 \\
\hline$>60$ & 23 & 8 \\
\hline \multicolumn{3}{|l|}{ Menopausal status } \\
\hline Unknown & 4 & 1 \\
\hline Premenopausal & 135 & 47 \\
\hline Perimenopausal & 26 & 9 \\
\hline Postmenopausal & 121 & 42 \\
\hline \multicolumn{3}{|l|}{ NACT groups } \\
\hline Group 1 & 148 & 52 \\
\hline Group 2 & 81 & 28 \\
\hline Group 3 & 57 & 20 \\
\hline \multicolumn{3}{|l|}{ Clinical stage } \\
\hline$\| \mathrm{A}$ & 14 & 5 \\
\hline IIB & 74 & 26 \\
\hline IIIA & 128 & 45 \\
\hline IIIB & 9 & 3 \\
\hline IIIC & 61 & 21 \\
\hline \multicolumn{3}{|l|}{ IHC-based subtype } \\
\hline Unknown & 42 & 15 \\
\hline Luminal-B & 27 & 9 \\
\hline Luminal-B/HER2+ & 35 & 12 \\
\hline HR-/HER2+ & 98 & 34 \\
\hline Triple-negative & 84 & 29 \\
\hline \multicolumn{3}{|l|}{$\mathrm{Ki}-67 \geq 40 \%$} \\
\hline Yes & 96 & 34 \\
\hline No & 150 & 52 \\
\hline Unknown & 40 & 14 \\
\hline \multicolumn{3}{|l|}{ NACT regimen } \\
\hline$A+T$ & 218 & 76 \\
\hline $\mathrm{PCb}$ & 20 & 7 \\
\hline $\mathrm{A}+\mathrm{T}+$ HER2-target & 27 & 9 \\
\hline $\mathrm{PCb}+\mathrm{HER} 2$-target & 21 & 7 \\
\hline
\end{tabular}

Table 1 (continued)
Table 1 (continued)

\begin{tabular}{|c|c|c|}
\hline Characteristics & Number of patients & $\%$ \\
\hline \multicolumn{3}{|l|}{ Sequence } \\
\hline Combination & 170 & 59 \\
\hline Sequential & 75 & 26 \\
\hline \multicolumn{3}{|l|}{ Schedule } \\
\hline Weekly chemotherapy & 36 & 13 \\
\hline 3-weekly chemotherapy & 250 & 87 \\
\hline \multicolumn{3}{|l|}{$\begin{array}{l}\text { Adjuvant endocrine } \\
\text { therapy }\end{array}$} \\
\hline Yes & 65 & 23 \\
\hline No & 221 & 77 \\
\hline \multicolumn{3}{|l|}{$\begin{array}{l}\text { Adjuvant radiation } \\
\text { therapy }\end{array}$} \\
\hline Yes & 77 & 27 \\
\hline No & 209 & 73 \\
\hline \multicolumn{3}{|l|}{ Outcomes } \\
\hline Progression & 35 & 12 \\
\hline $\begin{array}{l}\text { Death without } \\
\text { progression }\end{array}$ & 3 & 1 \\
\hline \multicolumn{3}{|l|}{ Site of metastasis } \\
\hline Distant first & 23 & 61 \\
\hline Brain & 10 & 26 \\
\hline Bone & 5 & 13 \\
\hline Liver & 2 & 5 \\
\hline Lung & 5 & 13 \\
\hline Mixed/other & 1 & 3 \\
\hline Local first & 12 & 32 \\
\hline Chest wall & 4 & 11 \\
\hline Ipsilateral breast & 4 & 11 \\
\hline Regional lymph node & 4 & 11 \\
\hline
\end{tabular}

IHC, immunohistochemical; HER, human epidermal growth factor receptor; HR, hormone receptor; NACT, neoadjuvant chemotherapy; $\mathrm{A}+\mathrm{T}$, adriamycin plus taxane; $\mathrm{PCb}$, paclitaxel plus carboplatin.

breast cancer $(5,17)$. Patients without pCR who receive intensive treatment may gain survival benefits; however, we aim to achieve individualized de-escalation of chemotherapy for patients with pCR. 


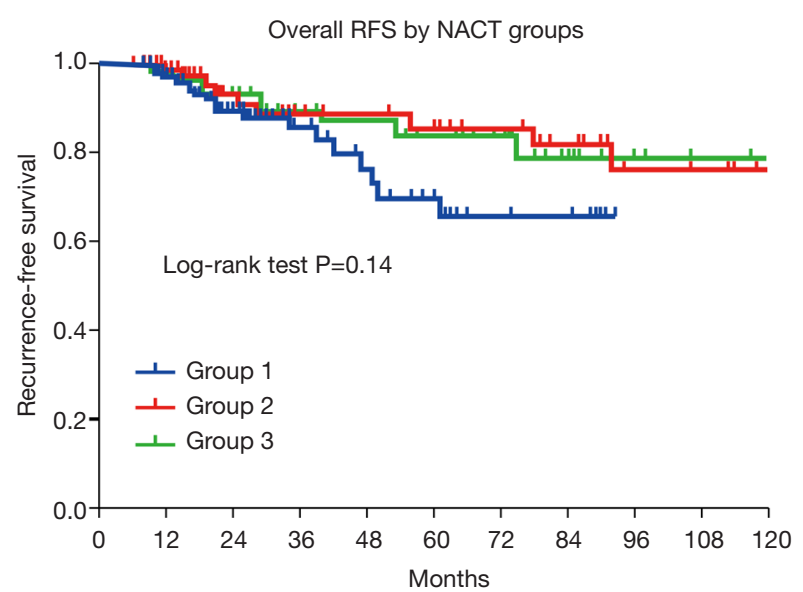

Figure 3 RFS for the entire cohort, compared among the three NACT groups. NACT, neoadjuvant chemotherapy; RFS, recurrence-free survival.

It is well established that pCR differs according to the therapeutic regimen and breast cancer subtype $(1,8,18)$, and the prognosis of patients with pCR is significantly better than that of patients without pCR (19). Previous retrospective studies have suggested that there is no significant difference in prognosis among different molecular types and NACT regimens in patients with pCR $(1,9,20)$. One meta-analysis by Spring et al. showed that, in patients who did or did not receive additional adjuvant chemotherapy after pCR, the 5-year event-free survival (EFS) rates were $86 \%$ and $88 \%$, respectively, and there was no significant difference $(\mathrm{P}=0.60)(8)$. However, in that study, the population that did not receive extra chemotherapy was described as "no more than $10 \%$ of patients were given postoperative intensive treatment", which is not the same as "no chemotherapy was given". Moreover, previous studies did not use image-guided biopsy to predict pCR. Further studies must be conducted to evaluate the effects of various levels of NACT de-escalation on the prognosis of patients who have achieved pCR.

The present study shows that the RFS of patients with pCR after NACT was not associated with the completion of standard chemotherapy cycles. Consistent with the results of Gonzalez-Angulo et al. (21), we observed that patients with stage IIIB and IIIC disease had a higher recurrence risk, even if they had achieved pCR. In addition, Gluz et al. reported that $90 \%$ of patients with Ki- 67 levels $\geq 40 \%$ were at high risk for recurrence (22). Hence, we conducted our retrospective analysis with a cut-off point of Ki-67
Table 2 Univariate analysis for factors correlated with survival

\begin{tabular}{|c|c|c|}
\hline Characteristics & $\begin{array}{l}\% \text { of patients with } \\
\text { events }(n=286)\end{array}$ & Log-rank P \\
\hline Age, years & & 0.33 \\
\hline $21-40$ & 10.3 & \\
\hline $41-60$ & 15.1 & \\
\hline$>60$ & 4.3 & \\
\hline Menopausal status & & 0.76 \\
\hline Premenopausal & 14.1 & \\
\hline Perimenopausal & 7.7 & \\
\hline Postmenopausal & 14.0 & \\
\hline Clinical stage & & $<0.0001$ \\
\hline$\|A+\| B$ & 5.7 & \\
\hline IIIA & 11.7 & \\
\hline $\mathrm{IIIB}+\mathrm{IIIC}$ & 25.7 & \\
\hline IHC-based subtype & & 0.10 \\
\hline Unknown & 14.3 & \\
\hline Luminal-B & 12.9 & \\
\hline HR-/HER2+ & 13.3 & \\
\hline Triple-negative & 13.1 & \\
\hline $\mathrm{Ki}-67 \geq 40 \%$ & & 0.069 \\
\hline Yes & 6.3 & \\
\hline No & 17.3 & \\
\hline Unknown & 15.0 & \\
\hline NACT regimen & & 0.75 \\
\hline$A+T$ & 14.2 & \\
\hline $\mathrm{PCb}$ & 10.0 & \\
\hline$A+T+$ HER2-target & 11.1 & \\
\hline $\mathrm{PCb}+$ HER2-target & 9.1 & \\
\hline Sequential & & 0.28 \\
\hline Combination & 17.6 & \\
\hline Sequential & 5.2 & \\
\hline Schedule & & 0.90 \\
\hline Weekly & 11.1 & \\
\hline 3-weekly & 13.6 & \\
\hline Adjuvant endocrine therapy & & 0.90 \\
\hline Yes & 10.8 & \\
\hline No & 15.4 & \\
\hline Adjuvant radiation therapy & & 0.36 \\
\hline Yes & 16.9 & \\
\hline No & 11.9 & \\
\hline
\end{tabular}

IHC, immunohistochemical; HER, human epidermal growth factor receptor; HR, hormone receptor; NACT, neoadjuvant chemotherapy; A + T, adriamycin plus taxane; $\mathrm{PCb}$, paclitaxel plus carboplatin. 
A
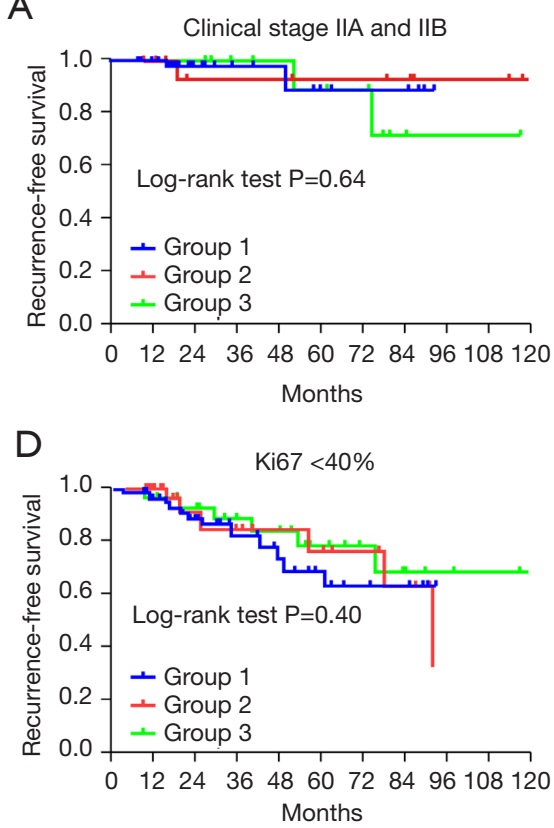

B

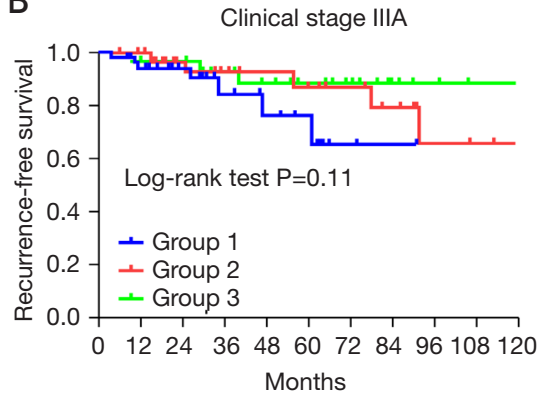

$\mathrm{E}$

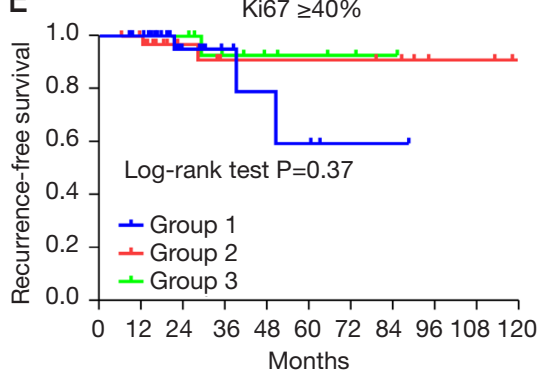

C
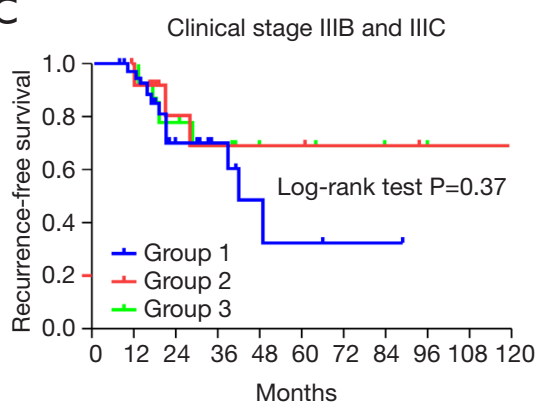

Figure 4 RFS by NACT groups within "high risk" patients. (A) RFS by NACT groups within clinical stage IIA + IIB; (B) RFS by NACT groups within clinical stage IIIA; (C) RFS by NACT groups within clinical stage IIIB + IIIC; (D) RFS by NACT groups within Ki- $67<40 \%$; (E) RFS by NACT groups within Ki-67 $\geq 40 \%$. NACT, neoadjuvant chemotherapy; RFS, recurrence-free survival.

Table 3 HRs of death, based on patient characteristics and treatments received

\begin{tabular}{lccc}
\hline Variables & Contrast & HR (95\% Cl) & P value \\
\hline NACT groups & Group 2 vs. group 1 & $0.74(0.29-1.89)$ & 0.53 \\
& Group 3 vs. group 1 & $0.55(0.23-1.31)$ & 0.18 \\
Age & $41-60$ vs. $\geq 60$ & $2.98(0.89-9.95)$ & 0.76 \\
& $20-40$ vs. $\geq 60$ & $1.21(0.13-11.79)$ & 0.87 \\
Ki-67 $\geq 40$ & Yes vs. no & $2.91(1.09-7.80)$ & 0.03 \\
Clinical stage & IIIA vs. IIA + IIB & $1.80(0.64-5.10)$ & 0.27 \\
& IIIB + IIIC vs. IIA + IIB & $5.13(1.80-14.58)$ & 0.002 \\
\hline
\end{tabular}

$\mathrm{HR}$, hazard ratio; $\mathrm{Cl}$, confidence interval; NACT, neoadjuvant chemotherapy.

levels of $40 \%$ and found that, even with pCR, patients with $\mathrm{Ki}-67 \geq 40 \%$ had a higher recurrence risk than those with $\mathrm{Ki}-67<40 \%$. Our data support the feasibility of terminating chemotherapy after achieving pCR, even in these high-risk patients.

Therefore, it is vital to assess the emergence of $\mathrm{pCR}$ in time. Due to the marked fibrosis after NACT and the changes in cell density and intraductal cancer after the disappearance of invasive cancer (23), ultrasonography and mammography cannot accurately assess residual tumors. For magnetic resonance imaging (MRI), recent studies have primarily used baseline levels of NACT and other parameters (e.g., tumor size, enhancement pattern, perfusion parameters obtained from dynamic contrast enhanced-MRI, and apparent diffusion coefficient values) to evaluate the sensitivity of NACT (24-27). Chemosensitivity to early NACT is then used to predict pCR; however, this approach has a low sensitivity $(28,29)$. In addition, there are no criteria for assessing pCR after NACT on MRI, and there are considerable limitations regarding non-mass 
enhancement and non-concentric shrinkage of tumors. Positron emission computed tomography can also be used to predict pCR (30); however, it nonetheless has defects in assessing pCR because it cannot detect lesions $<1 \mathrm{~cm}$ in diameter or distinguish reliably between benign and malignant tumors (31). Imaging-guided minimally invasive biopsy (MIB) has enormous potential for predicting pCR. In representative histological specimens of vacuum-assisted biopsy, the negative predictive value and false-negative rate are $94.4 \%$ and $4.8 \%$, respectively (32). Therefore, assessing whether to de-escalate chemotherapy under the guidance of MIB is very promising for individualized NACT.

Whether a single-institution cohort study is suitable for NACT patients at other institutions still needs to be confirmed. Our study has several limitations. First, although there was no statistically significant difference in RFS among patients in different NACT groups, the broad CI may not represent the true equality of the RFS, which may reflect the better prognosis of patients with pCR $(33,34)$, shorter follow-up times, and inadequate samples contributing to fewer end-point events (38/286). Second, among HER2-positive patients, only $36.1 \%$ received HER2-targeted therapy. Whether the use of targeted therapy affects survival in HER2-positive patients with pCR is unclear, with different studies showing conflicting results $(9,20,33)$. Third, the median follow-up times of groups one, two, and three were 20,22, and 51 months, respectively, which also reflected the NACT trend in our hospital. That is, after pCR achieved by NACT, fewer doctors and patients chose additional chemotherapy. Furthermore, the population distribution of the three groups was imbalanced because the baseline had biases. However, when we analyzed the differences in prognostic factors among the three groups, no significant differences were observed. Further prospective studies are needed to explore the safety of individual NACT de-escalation after pCR.

Despite these limitations, the multivariate analyses of this study revealed that baseline stage IIIB and IIIC disease and Ki-67 levels of $\geq 40 \%$ significantly increased the risk of recurrence of pCR patients compared to those without these factors. For these high-risk patients, our findings do not support further chemotherapy after pCR. NACT can be regarded as a sensitivity assessment for systemic therapy; patients with sensitive to NACT may further reduce their risk of recurrence. At present, we still cannot create a meaningful stratification of patients who achieved pCR. Achieving pCR is the best therapeutic outcome for patients, and additional systemic therapy will not significantly improve survival. Until the advent of more effective systemic therapeutic approaches, pCR can be regarded as the endpoint of NACT, which can be tailored and individualized.

\section{Conclusions}

Our results suggest that, even with pCR, patients with baseline stage IIIB and IIIC disease and Ki-67 levels of $\geq 40 \%$ had a higher recurrence risk than those without these factors. The RFS of patients with pCR was not associated with the completion of standard chemotherapy cycles, even in high-risk patients. Therefore, it is necessary to appropriately de-escalate chemotherapy in patients with pCR to reduce unnecessary toxicity and cost and avoid excessive treatment.

\section{Acknowledgments}

Funding: None.

\section{Footnote}

Conflicts of Interest: The authors have completed the ICMJE uniform disclosure form (available at http://dx.doi. org/10.21037/tcr.2020.01.01). The authors have no conflicts of interest to declare.

Ethical Statement: The authors are accountable for all aspects of the work in ensuring that questions related to the accuracy or integrity of any part of the work are appropriately investigated and resolved. All procedures performed involving human participants in our study were in accordance with the ethical standards of the Institutional and/or research committee (No. 201903801) and with the 1964 Helsinki Declaration and its later amendments or comparable ethical standards. Informed consent was obtained from all patients.

Open Access Statement: This is an Open Access article distributed in accordance with the Creative Commons Attribution-NonCommercial-NoDerivs 4.0 International License (CC BY-NC-ND 4.0), which permits the noncommercial replication and distribution of the article with the strict proviso that no changes or edits are made and the original work is properly cited (including links to both the formal publication through the relevant DOI and the license). See: https://creativecommons.org/licenses/by-nc-nd/4.0/. 


\section{References}

1. Cortazar P, Zhang L, Untch M, et al. Pathological complete response and long-term clinical benefit in breast cancer: the CTNeoBC pooled analysis. Lancet 2014;384:164-72.

2. Winer EP. Adjuvant and neoadjuvant therapy in patients with early breast cancer: Principles and practical considerations. The Breast 2019. doi: 10.1016/S09609776(19)30093-1.

3. Vaidya JS, Massarut S, Vaidya HJ, et al. Rethinking neoadjuvant chemotherapy for breast cancer. BMJ 2018;360:;5913.

4. Andreeva YY, Moskvina LV, Berezina TA, et al. Procedure for intraoperative material examination in breast cancer after neoadjuvant therapy to estimate residual cancer burden using the RCB system. Arkh Patol 2016;78:41-6.

5. Mittendorf EA, Vila J, Tucker SL, et al. The neo-bioscore update for staging breast cancer treated with neoadjuvant chemotherapy: incorporation of prognostic biologic factors into staging after treatment. JAMA Oncol 2016;2:929-36.

6. Masuda N, Lee SJ, Ohtani S, et al. Adjuvant capecitabine for breast cancer after preoperative chemotherapy. $\mathrm{N}$ Engl J Med 2017;376:2147-59.

7. von Minckwitz G, Huang CS, Mano MS, et al. Trastuzumab Emtansine for Residual Invasive HER2Positive Breast Cancer. N Engl J Med 2019;380:617-28.

8. Spring LM, Fell G, Arfe A, et al. Abstract GS2-03: pathological complete response after neoadjuvant chemotherapy and impact on breast cancer recurrence and mortality, stratified by breast cancer subtypes and adjuvant chemotherapy usage: individual patient-level meta-analyses of over 27,000 patients. American Association for Cancer Research 2019. doi: 10.1158/1538-7445.SABCS18GS2-03.

9. Weiss A, Bashour SI, Hess K, et al. Effect of neoadjuvant chemotherapy regimen on relapse-free survival among patients with breast cancer achieving a pathologic complete response: an early step in the de-escalation of neoadjuvant chemotherapy. Breast Cancer Res 2018;20:27.

10. Heil J, Sinn P, Richter H, et al. RESPONDER - diagnosis of pathological complete response by vacuum-assisted biopsy after neoadjuvant chemotherapy in breast Cancer a multicenter, confirmative, one-armed, intra-individuallycontrolled, open, diagnostic trial. BMC Cancer 2018;18:851.

11. van der Noordaa MEM, van Duijnhoven FH, Loo CE, et al. Identifying pathologic complete response of the breast after neoadjuvant systemic therapy with ultrasound guided biopsy to eventually omit surgery: Study design and feasibility of the MICRA trial (Minimally Invasive Complete Response Assessment). Breast 2018;40:76-81.

12. Goldhirsch A, Winer EP, Coates AS, et al. Personalizing the treatment of women with early breast cancer: highlights of the St Gallen International Expert Consensus on the Primary Therapy of Early Breast Cancer 2013. Ann Oncol 2013;24:2206-23.

13. Goetz MP, Gradishar WJ, Anderson BO, et al. NCCN guidelines insights: breast cancer, version 3.2018. J Natl Compr Canc Netw 2019;17:118-26.

14. Gnant $M$, Steger GG. Fighting overtreatment in adjuvant breast cancer therapy. Lancet 2009;374:2029-30.

15. Hayes DF. Targeting adjuvant chemotherapy: a good idea that needs to be proven! J Clin Oncol 2012;30:1264-7.

16. von Minckwitz G, Procter M, de Azambuja E, et al. adjuvant pertuzumab and trastuzumab in early HER2positive breast cancer. N Engl J Med 2017;377:122-31.

17. Selli C, Sims AH. Neoadjuvant therapy for breast cancer as a model for translational research. Breast Cancer (Auckl) 2019;13:1178223419829072.

18. Symmans WF, Wei C, Gould R, et al. Long-term prognostic risk after neoadjuvant chemotherapy associated with residual cancer burden and breast cancer subtype. J Clin Oncol 2017;35:1049-60.

19. Pelizzari G, Gerratana L, Basile D, et al. Post-neoadjuvant strategies in breast cancer: from risk assessment to treatment escalation. Cancer Treat Rev 2019;72:7-14.

20. Gonzalez-Angulo AM, Parinyanitikul N, Lei X, et al. Effect of adjuvant trastuzumab among patients treated with anti-HER2-based neoadjuvant therapy. Br J Cancer 2015;112:630-5.

21. Gonzalez-Angulo AM, McGuire SE, Buchholz TA, et al. Factors predictive of distant metastases in patients with breast cancer who have a pathologic complete response after neoadjuvant chemotherapy. J Clin Oncol 2005;23:7098-104.

22. Gluz O, Nitz UA, Christgen M, et al. West german study group phase III PlanB trial: first prospective outcome data for the 21-gene recurrence score assay and concordance of prognostic markers by central and local pathology assessment. J Clin Oncol 2016;34:2341-9.

23. Schaefgen B, Mati M, Sinn HP, et al. Can routine imaging after neoadjuvant chemotherapy in breast cancer predict pathologic complete response? Ann Surg Oncol 2016;23:789-95. 
24. O'Flynn EA, Collins D, D'Arcy J, et al. Multi-parametric MRI in the early prediction of response to neo-adjuvant chemotherapy in breast cancer: Value of non-modelled parameters. Eur J Radiol 2016;85:837-42.

25. Hylton NM, Blume JD, Bernreuter WK, et al. Locally advanced breast cancer: MR imaging for prediction of response to neoadjuvant chemotherapy--results from ACRIN 6657/I-SPY TRIAL. Radiology 2012;263:663-72.

26. Lee J, Kim SH, Kang BJ. Pretreatment prediction of pathologic complete response to neoadjuvant chemotherapy in breast cancer: perfusion metrics of dynamic contrast enhanced MRI. Sci Rep 2018;8:9490.

27. Tahmassebi A, Wengert GJ, Helbich TH, et al. Impact of machine learning with multiparametric magnetic resonance imaging of the breast for early prediction of response to neoadjuvant chemotherapy and survival outcomes in breast cancer patients. Invest Radiol 2019;54:110-7.

28. Gampenrieder SP, Peer A, Weismann C, et al. Radiologic complete response (rCR) in contrast-enhanced magnetic resonance imaging (CE-MRI) after neoadjuvant chemotherapy for early breast cancer predicts recurrencefree survival but not pathologic complete response (pCR). Breast Cancer Res 2019;21:19.

29. Telli ML. Insight or confusion: survival after response-

Cite this article as: $\mathrm{Li} \mathrm{X,} \mathrm{Liu} \mathrm{Y,} \mathrm{Shan} \mathrm{M,} \mathrm{Xu} \mathrm{B,} \mathrm{Lu} \mathrm{Y,} \mathrm{Zhang}$ G. Tailoring neoadjuvant chemotherapy for patients with breast cancer who have achieved pathologic complete response. Transl Cancer Res 2020;9(2):1205-1214. doi: 10.21037/tcr.2020.01.01 guided neoadjuvant chemotherapy in breast cancer. J Clin Oncol 2013;31:3613-5.

30. Wang Y, Zhang C, Liu J, et al. Is 18F-FDG PET accurate to predict neoadjuvant therapy response in breast cancer? A meta-analysis. Breast Cancer Res Treat 2012;131:357-69.

31. Heil J, Kümmel S, Schaefgen B, et al. Diagnosis of pathological complete response to neoadjuvant chemotherapy in breast cancer by minimal invasive biopsy techniques. Br J Cancer 2015;113:1565-70.

32. Heil J, Schaefgen B, Sinn P, et al. Can a pathological complete response of breast cancer after neoadjuvant chemotherapy be diagnosed by minimal invasive biopsy? Eur J Cancer 2016;69:142-50.

33. Huober J, Holmes E, Baselga J, et al. Survival outcomes of the NeoALTTO study (BIG 1-06): updated results of a randomised multicenter phase III neoadjuvant clinical trial in patients with HER2-positive primary breast cancer. Eur J Cancer 2019;118:169-77.

34. Sharma P, López-Tarruella S, García-Saenz JA, et al. Pathological response and survival in triple-negative breast cancer following neoadjuvant carboplatin plus docetaxel. Clin Cancer Res 2018;24:5820-9. 